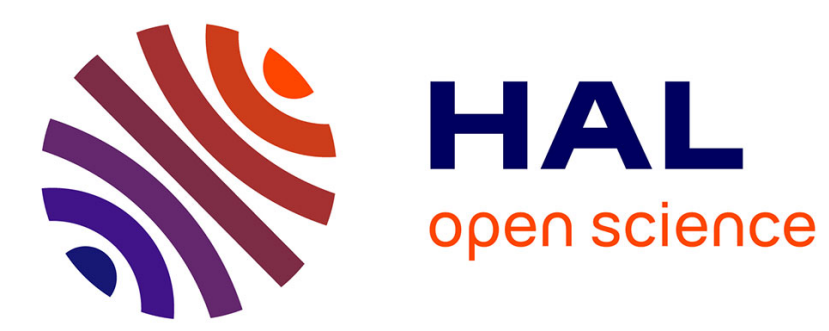

\title{
La bibliothèque Smith-Lesouëf à Nogent-sur-Marne, une fondation bien particulière
}

Isabelle Duhau

\section{To cite this version:}

Isabelle Duhau. La bibliothèque Smith-Lesouëf à Nogent-sur-Marne, une fondation bien particulière. Livraisons d'Histoire de l'Architecture, 2006, 11 (1), pp.33 - 50. 10.3406/lha.2006.1031 . hal-01858847

\section{HAL Id: hal-01858847 \\ https://hal.science/hal-01858847}

Submitted on 21 Aug 2018

HAL is a multi-disciplinary open access archive for the deposit and dissemination of scientific research documents, whether they are published or not. The documents may come from teaching and research institutions in France or abroad, or from public or private research centers.
L'archive ouverte pluridisciplinaire HAL, est destinée au dépôt et à la diffusion de documents scientifiques de niveau recherche, publiés ou non, émanant des établissements d'enseignement et de recherche français ou étrangers, des laboratoires publics ou privés. 


\section{La bibliothèque Smith-Lesouëf à Nogent-sur-Marne, une fondation} bien particulière

Isabelle Duhau

\section{Citer ce document / Cite this document :}

Duhau Isabelle. La bibliothèque Smith-Lesouëf à Nogent-sur-Marne, une fondation bien particulière. In: Livraisons d'histoire de l'architecture, $\mathrm{n}^{\circ} 11$, 1er semestre 2006. pp. 33-50;

doi : https://doi.org/10.3406/lha.2006.1031

https://www.persee.fr/doc/lha_1627-4970_2006_num_11_1_1031

Fichier pdf généré le 02/04/2018 


\title{
Résumé
}

"La bibliothèque Smith-Lesouëf à Nogent-sur-Marne, une fondation bien particulière ", par Isabelle Duhau La très riche bibliothèque d'Auguste Lesouëf (1829-1906) n'est installée dans une architecture appropriée qu'après sa mort. Ses héritières, ses deux nièces Jeanne et Madeleine Smith, offrent l'ensemble de la collection à l'État, à la condition que celle-ci reste conservée dans un édifice qu'elles décident de faire construire pour l'accueillir, à Nogent-sur-Marne, au cœur de la propriété qu'elles y possèdent. Ce faisant, elles réussissent à sauvegarder leur vaste domaine, menacé par des aménagements urbains. La bibliothèque, bâtie entre 1913 et 1916 par l'architecte Théodore Dauphin, tente une difficile synthèse entre les exigences complexes du programme et les choix décoratifs personnels des commanditaires, les deux sœurs, mais aussi et surtout, I'historien Pierre Champion, fils de l'éditeur Honoré Champion et mari de Madeleine. Tout à la fois bibliothèque et musée, bibliothèque privée et bibliothèque publique, bibliothèque savante et bibliothèque généraliste, la fondation Smith-Lesouëf se présente encore comme une entité exceptionnelle ; car si l'histoire des bibliothèques privées françaises sous l'Ancien Régime n'est plus à faire, celle des institutions publiques au XIXe puis au XXe siècle a peut-être occultée une histoire des collections des bibliophiles de cette période qu'il reste à enrichir.

\begin{abstract}
"The Smith-Lesouëf's library in Nogent-sur-Marne. Such an exceptional fundation", by Isabelle Duhau. Only after his death is Auguste Lesouëf s very rich library settled in a specific architecture. His two heiresses and nieces, Jeanne and Madeleine Smith, offer the whole collection to the French State, on condition that it remains kept in a building they decide to have erected in Nogent-sur-Marne, in the heart of a land of their own. Meanwhile they succeed in saving their property which was threatened by urban plannings. The library, built between 1913 and 1916 by the architect Théodore Dauphin, was designed as a combination of the complex planning requirements and the decorative choices of not only the two commissionning sisters, but also of the historian Pierre Champion, the publisher Honoré Champion's son and Madeleine's husband. All together a library and a museum, a private and a public library, a scholarly and a general-interest library, the Smith-Lesouëf s fundation appears as an exceptionnal entity ; for if everybody knows quite well about Ancien Régime French private libraries, the history of the XIXth and XXth centuries public institutions may have overshadowed a history of bibliophiles'collections of this period that remains to be enriched.
\end{abstract}

\section{Zusammenfassung}

"Die Privatbibliothek Smith-Lesouëf in Nogent-sur-Marne, eine ganz besondere Stiftung ", von Isabelle Duhau Die sehr reiche Bibliothek des August Lesouëf (1829-1906) wird erst nach dessen Tod in einer geeigneten Architektur untergebracht. Die Erbinnen, beziehungsweise seine zwei Nichten, Jeanne und Madeleine Smith, schenken die gesamte Sammlung dem Staat, unter der Bedingung, dass sie in einem Gebäude aufbewahrt werde, das sie mitten in ihrem eigenen Grundbesitz in Nogentsur-Marne bauen lassen wurden. Dadurch gelingt es ihnen gleichzeitig ihr ausgedehntes Gebiet vor der urbanen Planung zu retten. Der Architekt Théodore Dauphin baut die Bibliothek zwischen 1913 und 1916. Er ubernimmt die schwere Aufgabe, die komplexen Bedingungen des Programme mit den dekorativen Ansprtichen der Auftraggeberinnen, der zwei Schwestern einerseits, aber auch und hauptsächlich, derer des Historikers Pierre Champion, Sohn des Verlegers Honoré Champion und Madeleines Ehemann abzuwägen. Gleichzeitig Bibliothek und Museum, private und öffentliche Bibliothek, Allgemein- und Gelehrtenbibliothek erweist sich die Stiftung Smith-Lesouëf noch als eine aufierordentliche Einrichtung. Zwar ist die Geschichte der franzôsischen Privatbibliotheken unter dem Ancien Régime gut bekannt, aber in der Geschichte der öffentlichen Institutionen im 19. und 20. Jahrhundert verbirgt sich auch eine Geschichte der Sammlungen der Bibliophilen zu dieser Zeit, die noch zu ergänzen ist.

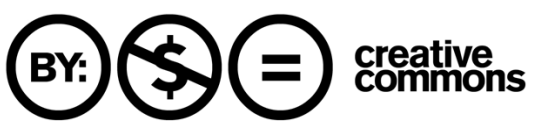




\title{
LA BIBLIOTHÈQUE SMITH-LESOUËF À NOGENT-SUR-MARNE, UNE FONDATION BIEN PARTICULIËRE
}

\begin{abstract}
Enfant, j'espérais devenir un livre quand je serais grand. Pas un écrivain, un livre : les hommes se font tuer comme des fourmis. Les écrivains aussi. Mais un livre, même si on le détruisait méthodiquement, il en subsisterait toujours quelque part un exemplaire qui ressusciterait sur une étagère, au fond d'un rayonnage dans quelque bibliothèque perdue...

Amos $\mathrm{Oz}$, Une Histoire d'amour et de ténèbres.
\end{abstract}

Selon le Grand Robert de la langue française, une bibliothèque est tout à la fois une collection de livres, un meuble dont les tablettes permettent de ranger et de classer des livres et une salle ou un édifice dans lequel sont placés des livres pouvant être consultés. Le plus souvent, les bibliothèques d'érudits réunissent ces trois définitions. Leur évocation renvoie à la pièce spécifique d'une demeure, garnie de sombres rayonnages de bois du sol au plafond, sur lesquels s'alignent dans la pénombre et le silence des milliers d'ouvrages reliés plein cuir; le long d'une paroi, une échelle, au centre, un bureau massif, éclairé d'une lampe, où travaille l'honnête homme.

Alexandre-Auguste Lesouëf (1829-1906) consacra sa vie et sa fortune à réunir une collection de livres, d'estampes et de manuscrits. Ce n'est pourtant pas à lui que revient la paternité de l'édifice de Nogent-sur-Marne, à proximité immédiate de Paris, construit et aménagé spécifiquement pour y transporter sa bibliothèque, mais à ses héritières, sa sœur Anne-Léontine Smith-Lesouëf et ses nièces Jeanne et Madeleine Smith, et peut-être encore davantage au mari de cette dernière, l'historien Pierre Champion. Ainsi, la fondation Smith-Lesouëf ne se présenta jamais ni comme une bibliothèque strictement privée, la collection fut offerte à l'État en 1913, ni comme une véritable bibliothèque publique : le bâtiment construit par l'architecte Théodore Dauphin pour l'abriter, n'était ouvert que quelques heures par semaine et resta en quelque sorte une annexe de la demeure des SmithChampion (ill. 1).

L'histoire de cet ensemble mérite d'être retracée : non seulement l'édifice apparaît comme un exemple architectural très isolé, mais la collection qu'il contenait a quitté Nogent pour les rayonnages de la Bibliothèque nationale de France ${ }^{1}$.

1. Nous avons étudié la bibliothèque Smith-Lesouëf dans le cadre d'un travail conduit entre 2004 et 2005 au sein du service de l'Inventaire général du patrimoine culturel de la direction régionale des affaires culturelles d'lle-de-France. Ces recherches ont conduit à une publication dont plusieurs pages sont consacrées aux propriétés nogentaises de la famille Smith-Champion : Inventaire général 


\section{La fondation : une réponse aux aménageurs}

Auguste Lesouëf ${ }^{2}$, rentier, héritier d'un père affineur d'or et d'argent, est un homme dont

on est surpris qu'avec des ressources en somme limitées, il ait formé une collection aussi précieuse, qui est comme le petit Chantilly ${ }^{3} \mathrm{~d}^{\prime}$ un bourgeois de Paris. [...] 250 manuscrits et recueils d'autographes (et quels manuscrits !), 18000 volumes [...], 35 incunables; 171 volumes du XVI ${ }^{e}$ siècle; les principaux auteurs français du XVII siècle dans les éditions originales; une série parfois aux armes, des grands livres illustrés du XVIII' siècle, une suite des entrées royales; une importante bibliothèque thêâtrale; une magnifique série de livres sur les costumes, parfois avec les dessins originaux; les principaux recueils de la topographie de l'ancienne France; une collection, aussi rare que précieuse, sur l'histoire de Paris ; une autre, importante, de mémoires; une bibliothèque américaine, précolombienne, chinoise et japonaise [...]. Il ne faut pas oublier une série d'auteurs modernes [...]. Enfin, Auguste Lesouëf avait réuni sur Paris une série importante de plans, de gravures de mœurs. Il fit même faire par Chaumet une suite de dessins et d'aquarelles représentant les aspects du vieux Paris, ses démolitions. Enfin, il a constitué sur l'histoire de France une suite d'estampes ${ }^{4}[. .$.$] . Il faut ajouter$ à cela un fonds admirable de peintures chinoises et japonaises, de miniatures persanes, un médaillier précieux, une collection de jeux anciens, des armes, des modèles de construction et de navires, des ivoires, des netzukis', quelques figures antiques et tant de perites poupées ${ }^{6}$.

Lesouëf est un client du fameux libraire et éditeur parisien, Honoré Champion. Il se lie d'amitié avec l'un des fils de la maison, Pierre (1880-1942). En 1904, il aide ce dernier, encore élève à l'École des chartes, à financer l'édition d'un premier ouvrage $^{7}$. L'année suivante, le jeune homme entreprend le catalogue des manuscrits

du patrimoine culturel, région Ile-de-France. Dominique Hervier (dir.), Isabelle Duhau (réed.), Stéphane Asseline et Laurent Kruszyk (photogr.), Nogent et Le Perreux, l'Eldorado en bord de Marne. Paris, APPIF, 2005, 144 p.

2. Véronique Béranger, Japonisme et Érudition: Le livre japonais dans les collections d'Auguste Lesouëf (1829-1906), thèse de l'École des Chartes, 2000. Nous tenons à remercier Véronique Béranger qui, par sa connaissance du fonds Smith-Lesouëf de la BnF, nous en a facilite l'acces, ainsi que Mme Le Pavec, conservateur au département des manuscrits, qui a réalisé l'inventaire des archives du fonds et nous les a communiquées.

3. En reférence à la collection du duc d'Aumale, la bibliothèque de l'actuel musté Condé.

4. L'inventaire en dénombrera 17491.

5. Netsuke: dans le costume traditionnel japonais, figurine le plus souvent en ivoire, servant de contrepoids aux objets attachés à la ceinture.

6. "Pierre Champion, Auguste Lesouëf, collectionneur", La Gerbe, 22 janvier 1942.

7. Il s'agit d'un recueil de fac-similés publiés par Jacques Monfrin, Les Plus anciens monuments de la typographie parisienne. Honore Champion et sa librairie. 1874-1978, Paris, ed. Honoré Champion, 1978, p. 107. 
de Lesouëf ${ }^{8}$. À la fin de sa vie, le collectionneur solitaire ne fréquente plus guère que sa sœur et ses deux nièces qui héritent de ses biens, actions et immeubles, mais aussi de sa formidable collection, entassée dans son appartement parisien du boulevard Beaumarchais, lorsqu'il meurt en 1906.

Les trois femmes ne fréquentent pas davantage le monde. Elles résident le plus souvent à Nogent-sur-Marne dans une vaste propriété constituée de deux demeures de plaisance datant de l'Ancien Régime, contiguës (14 et 16, avenue Charles VII) dont les jardins s'étageant sur le coteau dominant la Marne ont été réunis pour former un parc de plusieurs hectares. Il semble que les deux jeunes filles aient reçu une éducation sévère. Madeleine (1864-1940) est peintre. Élève et intime de JeanJacques Henner, elle vit recluse et désenchantée depuis que celui-ci a refusé de l'épouser malgré sa promesse, puis est mort en 1905'. Jeanne (1857-1943), qui s'adonne à la photographie, est toujours restée auprès de sa mère.

Au décès de Lesouëf, c'est tout naturellement Pierre Champion qui est chargé de l'inventaire des collections. Durant la première moitié de l'année 1907, il travaille presque chaque après-midi boulevard Beaumarchais, aidé par Madeleine. Ainsi, malgré les seize années qui les séparent, naît un véritable attachement entre ces deux êtres, mélange d'estime intellectuelle, de passion pour l'étude et la culture et de reconnaissance mutuelle pour la vie sereine qu'ils s'offrent l'un à l'autre. Ils se marient le 19 décembre et partagent désormais leur temps entre Paris, Nogent et de nombreux voyages.

Bientôt, la propriété nogentaise est menacée. La municipalité envisage de percer un boulevard pour désengorger le centre ville, qui traverserait le parc de part en part (ill. 2). Les Smith-Champion réussissent à contrecarrer le projet en obtenant en $1909^{10}$ le classement du domaine au titre des sites. Madeleine est viscéralement attachée à ce paysage du bord de la Marne qu'elle peint régulièrement. Son souhait le plus vif est de le conserver intact à tout jamais. Ainsi naît dans l'esprit de cette famille, sans descendance, l'un des arguments décisifs de la protection du lieu, l'engagement qu'elle réalise de donner le domaine à l'État et d'y créer un muséebibliothèque autour des collections d'Auguste ${ }^{11}$. Jean Babelon, conservateur du cabinet des médailles, informe l'administrateur général de la Bibliothèque nationale de l'éventuelle donation :

8. Véronique Béranger, op. cit. Cet inventaire sera publié en 1930.

9. BnF, dép. des mss., fonds Smith-Lesouëf (don 36480), carton 29, journal intime de Madeleine, 1906-1907.

10. Arrêté de classement du 19 février 1909.

11. Champion apporta un autre argument. Il invoqua la mémoire du peintre Antoine Watteau, décédé en 1721 dans cette maison et dont le parc aurait inspiré les dernières ceuvres. Il reconnut après coup s'être trompé sur l'interprétation de certaines sources car Watteau était en réalité mort dans la propriété voisine. Ce que certains considérèrent comme une « surprenante " erreur pour un historien de son envergure atteint finalement son but puisque l'abandon du projet de boulevard sauva aussi cet autre parc, devenu aujourd'hui un jardin public. 
Ill. 2: Plan de la propriété du 14 et 16 avenue Charles VII à Nogent-sur-Marne (FNAGP). Ce plan fut dressé à l'appui de l'argumentaire de la famille Smith-Champion au moment de la polémique sur le futur boulevard et diffusé sous forme phorographique à toutes les personnalités d'influence qu'elle rencontra à l'occasion de sa croisade. Fn bas à droite, en blanc, l'implantation de la future bibliothèque entre les deux demeures. Inventaire général. C.. S. Asseline, ADAGP.

C'est pour parer à cette éventualité [le boulevard] que les propriétaires [...] proposent de donner à l'État l'immeuble et les collections, sous la seule condition que l'avenue projetée ne serait pas faite. [...] Je ne fais que traduire et résumer une longue conversation que j’ai eue avec Madame Smith [...]. Dans tous les cas, je me permets de rappeler la conclusion de mon rapport antérieur : c'est que les collections Lesouëf sont scientifiquement et artistiquement de la plus haute importance et que la proposition de donation me paraît mériter un sérieux examen ${ }^{2}$.

12. BnF, archives administratives de la donation Smith-Lesouëf, dossier E87/20. 
La commission des sites rejette définitivement le projet de boulevard en $1912^{13}$; les négociations ne sont pas achevées lorsque Mme Smith décède et il revient à ses deux filles de contracter avec l'État, qui accepte, par décret du 30 juillet 1913, la « libéralité » [...] pour la Bibliothèque nationale :

$1^{\circ}$ d'une bibliothèque ;

$2^{\circ}$ d'une collection de monnaies, statuettes, etc. ;

$3^{\circ}$ de tableaux de Henner et Raphaël Collin ${ }^{14}$;

$4^{\circ}$ d'un terrain d'une superficie de cent trente quatre mètres dix huit centièmes, sis à Nogent-sur-Marne ;

$5^{\circ}$ d'une somme de deux cent cinquante mille francs (250 000 francs.) dont les revenus serviront à faire face aux dépenses de toutes sortes nécessitées par le service et l'entretien de la Bibliothèque et des collections;

$6^{\circ}$ d'une somme de cent cinquante mille francs (150000 francs) destinée à faire élever sur le terrain sus-désigné une construction qui renfermera la Bibliothèque, les collections et les tableaux.

Cependant, la délivrance des legs est consentie aux charges et conditions suivantes :

$1^{\circ}$ La bibliothèque, les collections et les tableaux devront rester à perpétuelle demeure à Nogent-sur-Marne, dans le bâtiment qui leur est destiné [...] ; $2^{\circ}$ Mlle Smith et Mme Champion se réservent [...] de proposer à la nomination de M. le Ministre [...] le conservateur de la bibliothèque et des collections $[. .$.$] ; le garçon qui sera attaché au service de la bibliothèque [...]$ devra être agréé par elles [...] ;

$5^{\circ}$ la bibliothèque sera ouverte au public comme musée, tous les dimanches et jours de fêtes. [...] Une salle publique [...] sera ouverte plusieurs jours de la semaine sous la surveillance du gardien et du bibliothécaire ${ }^{15}$.

Le bâtiment projeté est construit entre 1913 et 1916. Parallèlement, durant la première guerre mondiale, Madeleine et Jeanne transforment leurs maisons nogentaises en hôpital ${ }^{16}$, tandis que Pierre passe les deux premières années au front, puis est envoyé à Rabat, attaché au secrétariat général du Protectorat ${ }^{17}$. À son retour, terriblement marqué, il n'envisage pas de reprendre le cours de sa vie passée et décide de s'engager dans la vie publique; il est élu maire de Nogent dès 1919 et le restera jusqu'à sa mort. Le couple vit désormais la plus grande partie de l'année à Nogent. C'est là que Champion partage son temps entre la mairie et ses travaux

13. Loc. cit., carton 40, lettres de Jeanne à sa sour, 1908-1912.

14. Ces cuvres appartiennent au couple Champion qui les ajoutent à la collection Lesouëf.

15. Loc. cit., dossier E87/2.

16. Elles accueillent jusqu'à soixante-dix malades dans "l'hôpital auxiliaire 73 ".

17. Loc. cit., carton 36, lettres de Pierre à Madeleine. Juillet 1916-décembre 1918. 
de recherches, qu'il installe sa propre bibliothèque ${ }^{18}$ tandis que sa femme peint dans son atelier. Madeleine meurt la première, le 18 avril 1940. Elle lègue par testament à l'État français le 16, rue Charles VII [qui] devra être conservé dans son état actuel comme annexe de la fondation Smith-Lesouëf (14 bis, rue Charles VII) et transformé en musée :

On répartira ce qui n'a pu être exposé de la collection de notre oncle M. Auguste Lesouëf [...] ; on y installera mes meubles anciens, les meilleurs de mes tableaux et tous objets dignes d'être gardés dans un musée. Je désire que le parc attenant à cette maison d'une contenance de cinq hectares soit conservé dans son état actuel [...] et qu'il soit ouvert au public [...]. Quand à la propriété de cinq hectares, 14, rue Charles VII que je possède en indivision avec ma soeur et qu'elle habite, notre désir est qu'elle soit affectée à une maison de retraite pour des artistes et des écrivains des deux sexes. [...] Je lègue à mon mari tout notre mobilier de Paris à l'exception de mes bijoux, des six fauteuils de tapisserie Louis XVI, du petit bureau et des tapisseries perlées qui se trouvent dans le petit salon, et des deux esquisses de Henner qui sont dans le salon, objets devant être installés dans le musée de Nogent ${ }^{19}$.

Champion s'éteint le 29 juin $1942^{20}$ et Jeanne, quelques mois plus tard ${ }^{21}$. Jusqu'à leur décès, la fondation Smith-Lesouëf vit de fait sous la double tutelle de l'Etat et du couple Smith-Champion. Elle est implantée au cœur de leur propriété, ils y demeurent " chez eux $"^{22}$.

18. Archiviste palégraphe, Champion est un historien spécialiste du XVe siècle. Il édite des manuscrits et publie des biographies de Charles d'Orléans, de François Villon, de Jeanne d'Arc, d'Agnès Sorel, une histoire de France, une histoire de la potsie du XV' siècle, etc. ; il devient également historien de sa terre d'adoption, Nogent, où Charles V fit construire le manoir de Beauté que Charles VII donna à sa maîtresse, Agnès Sorel, d'où son surnom de dame de Beauté... Il reçoit plusieurs prix pour ses écrits jusqu'à entrer à l'Académie des sciences morales et politiques en 1940, puis à l'Académie Goncourt en 1941.

19. Arch. mun., Nogent-sur-Marne, 2L2/4.

20. Il offre par testament une partie de sa bibliothèque à la ville de Nogent-sur-Marne, à celle de Châtenay, aux Amis de Vincennes et à la bibliothèque de l'Institut, tandis qu'une autre partie est dispersée en vente publique (voir le catalogue de la vente: Edouard Giard, commissaire-priseur; Georges Andrieux, expert près les douanes françaises. Bibliotheque Pierre Champion, 26 et 27 fevrier 1945, hôtel Drouot, salle 9, 47 p.). Une partie de la bibliothèque sera racheté en 1965 par les futurs acquéreurs des éditions Honoré Champion. Jacques Monfrin, op. cit., p. 7.

21. Jeanne meurt le 23 avril 1943, elle confirme dans son propre testament les dispositions de sa soeur et ajoute le don de ses meubles et objets d'art qui doivent rejoindre le musée. Arch. mun., Nogentsur-Marne, 2L2/4.

22. Pierre Champion est même nommé conservateur honoraire de la bibliothèque de 1925 à sa mort. Loc. cit., dossier E87/31. 


\section{La fondation: un chantier dans la tourmente}

Puisque la fondation Smith-Lesouëf doit conforter l'intégrité du domaine nogentais, il faut lui bâtir sur place un écrin digne d'elle. Sans expérience en la matière, les héritières font appel à Théodore Dauphin, l'architecte de leur oncle, qui est associé à son gendre Paul-Marion ${ }^{23}$. Dauphin (1849-1917), second grand prix de Rome en 1878, architecte en chef des bâtiments civils (chargé de l'École de pharmacie), est un digne représentant de l'École des beaux-arts. Lauréat de nombreuses médailles et récompenses, il a construit, outre des immeubles de rapport parisiens, l'École supérieure de pharmacie d'Alger, l'observatoire zoologique maritime de Tatihou et plusieurs édifices pour la famille du peintre Ernest Meissonier ${ }^{24}$. Fin 1913, il élabore le projet qui doit être achevé en une année et qui est approuvé par le ministre de l'Instruction publique et des beaux-arts ${ }^{25}$ (ill. 3).

Ill. 3 : Vue extérieure d'ensemble de la bibliothèque, tirage photographique, vers 1920 . BnF.

23. Dauphin était notamment chargé de l'entretien des immeubles d'Auguste Lesouëf. Le papier à lettres de l'agence, installé 7, rue Royale à Paris, précise pour Dauphin, * architecte du gouvernement, expert près le tribunal civil » et pour Marion, " architecte diplômé (PLG) ». Loc. cit., carton 14, documents concernant les biens d'Auguste Lesouëf.

24. Edmond Delaire. Les Architectes elezves de l'École des beaux-arts 1793-1907, Paris, librairie de la Construction moderne, 1907, $2^{\text {e }}$ édition. Ministère de la culture, bases de données Archidoc et Mérimée. Michel Fleury (dir.), Anne Dugast et Isabelle Parizet (rét.), Dictionnaire par noms d'architectes des constructions elevées a Paris aux XIX'et $X X^{e}$ sizcles. II, Dabernat dे Guyran : première série, période 1876-1899 [....] Paris, service des travaux historiques, 1991. Centre de documentation du musfe d'Orsay, section architecture. Nous remercions Isabelle Loutrel.

25. Loc. cit., dossier E87/18. 
Très rapidement, le chantier commence. Les fondations sont creusées avant la fin de $1913^{26}$. Mais la guerre perturbe les travaux qui vont se poursuivre lentement jusqu'à leur achèvement en 1916. Madeleine, restée sur place, assume seule la maîtrise d'ouvrage, guidée par son mari depuis le fond de sa tranchée. Ils échangeront durant le conflit des centaines de lettres; cette correspondance, témoignage intime émouvant sur cette période tragique, rapporte de nombreux détails sur le déroulement du chantier, les choix architecturaux et ceux des décors ${ }^{27}$. Madeleine s'en remet aux avis de son époux et ne prend aucune décision sans l'avoir consulté. Il est à ses yeux le spécialiste de la question, mais elle réussit aussi par ce biais à l'abstraire quelques instants de sa condition en le transportant en esprit au cour de sa passion : les livres ${ }^{28}$.

En octobre 14, tandis que le gros-œuvre est bien avancé, Pierre envoie une carte postale du château de Dury ${ }^{29}$, village situé près d'Amiens, afin que les ouvriers s'en inspirent pour achever le toit (ill. 4). Quelques mois plus tard, le 6 août 1915, sa femme l'informe de la situation:

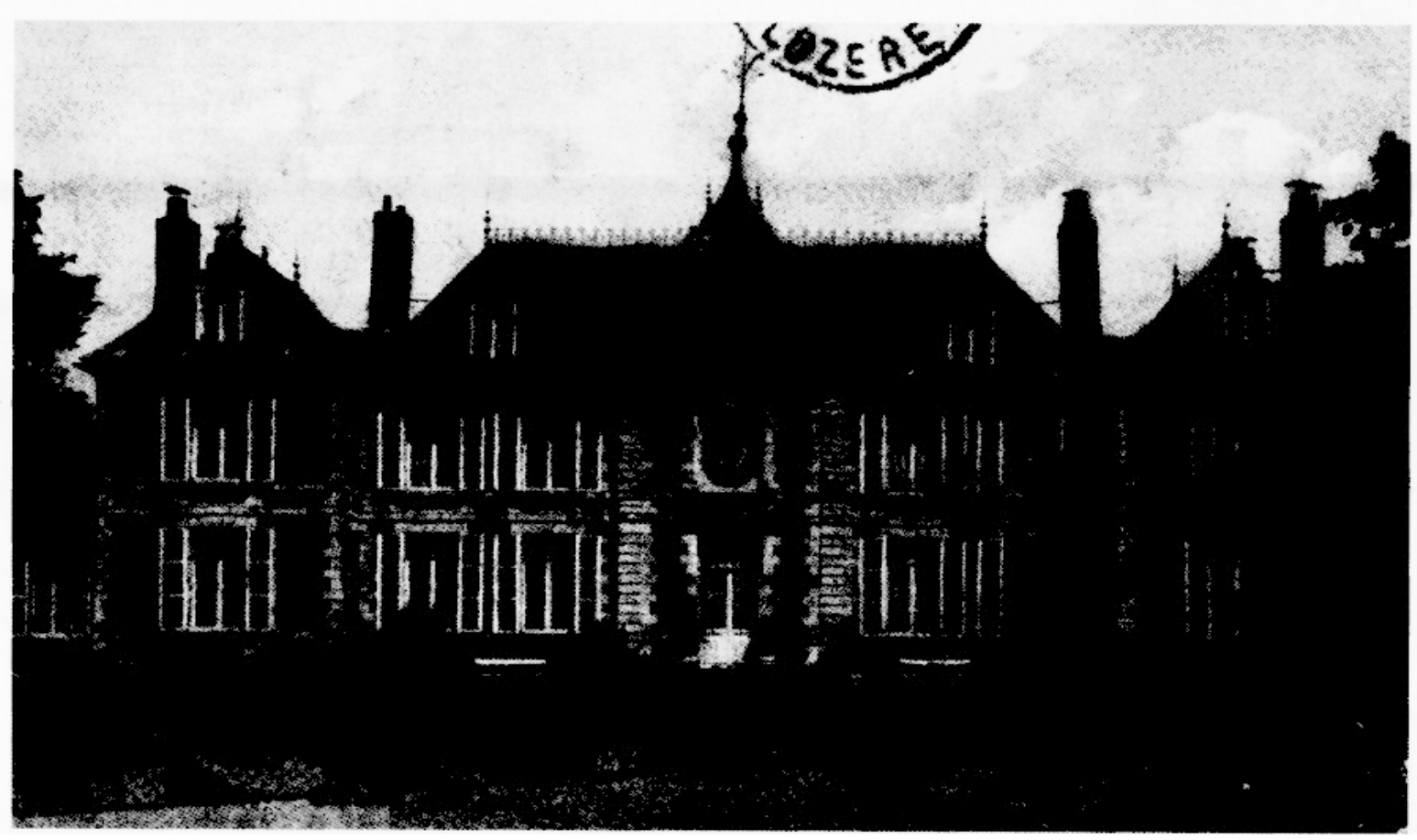

Ill. 4 : Façade sud du château de Dury. Carte postale, vers 1910, coll. part.

26. Loc. cit., carton 41, lettres de Jeanne à sa scur. 1913.

27. Cartons 30-31-32, lettres de Madeleine à Pierre, aoât 1914/juin 1915 - juillet 1915/décembre 1916 - 1917/1919. Cartons 33-34-35-36, lettres de Pierre à Madeleine, août 1914/juillet 1915 août/déc. 1915 - janvier/juin 1916 - juillet 1916/décembre 1918.

28. Elle fait son possible pour qu'il continue de travailler, en lui faisant parvenir des ouvrages, en transcrivant les textes qu'il lui envoie, en effectuant elle-même certaines recherches, en entretenant des contacts avec son réseau d'érudits... tandis que Champion, de son côté, l'encourage à ne pas abandonner la peinture malgré la charge de l'intendance de l'hôpital.

29. Le château aurait été construit au XVIII siècle. Il fut occupé par l'armée durant la grande guerre puis détruit en 1940 lors d'une offensive allemande. Nous remercions Pascale Touzet, de la conservation régionale des monuments historiques de Picardie, pour ces informations. 
Ici le toit est déjà fini ; il est à mon avis un peu trop droit, pas assez incliné ; les architectes modernes ne veulent pas admettre la forte inclinaison des vrais toits anciens, sans doute dans le but utilitaire de gagner de la place à l'intérieur. Tel qu'il est cependant, notre construction a bonne apparence, la lucarne, dont M. Mallet a exécuté la sculpture, est très jolie, les colonnes de la petite tourelle, sont aussi fort élégantes. [...] Si nous pouvions avoir de toi les indications indispensables pour la disposition des rayons des bibliothèques, on pourrait peut-être commencer l'aménagement intérieur.

Dauphin, déjà très malade, ne peut se rendre sur le chantier que de loin en loin, et son gendre, soldat, ne vient à Nogent qu'à l'occasion de rares permissions ; aussi, peu à peu, l'entrepreneur de maçonnerie, Rameau, prend-il plus d'importance auprès de Madeleine. Tandis que l'architecte dessine chaque élément du décor qui reste à fabriquer, Rameau emmène sa cliente chiner des pièces anciennes qu'il remonte et adapte à leur nouveau contexte. Ainsi, après avoir soumis des croquis ou des photographies à Pierre, celle-ci achète un balcon pour l'une des salles de la bibliothèque et des panneaux de portes à deux vantaux, l'ensemble provenant de la démolition de l'hôtel des Conseils de guerre, rue du Cherche-Midi ${ }^{30}$. Elle acquiert également, pour la balustrade de la grande salle, six panneaux qui serviront de motifs pour chacun des côtés de la salle, qui proviennent, eux, de la démolition de l'ancien couvent des Filles de l'Assomption, rues Cambon et Saint-Honorér ${ }^{11}$; avant de conclure: "Que c'est triste de voir ces débris artistiques au milieu de ferraille, de toutes sortes, de choses innommables! Mais je crois qu'en cherchant un peu nous pourrons arriver à une sérieuse économie, et obtenir un bien meilleur résultat qu'en laissant M. D. exécuter ses dessins. "Durant le premier trimestre de 1916, les sols sont en cours, dallage noir et blanc, selon les pièces, ou parquets copiés sur les sols anciens de la demeure, après que Madeleine a soigneusement déterminé avec le fumiste les emplacements des radiateurs pour préserver au mieux les livres. Reste la confection des rayonnages. Tandis que les architectes proposent dans un premier temps un dessin prévoyant, en partie basse, des avant-corps destinés aux in-folio, surmontés de tablettes moins profondes, Champion écrit : "Cela me ferait plaisir d'avoir une petite idée du dessin de M. Marion pour la bibliothèque. Fais une petite photo si c'est possible. J'avoue que de penser aux livres, ça m'attendrit toujours un peu. Bon Dieu, quels sauvages nous sommes $!^{32}$ "Après avoir reçu le dessin, dans une autre lettre, il commente : "J'ai vu aussi les croquis de la bibliothèque, fais pour le mieux. Mais pourquoi ne pas faire courir l'avance

30. Cet hôtel, ancien l'hôtel de Toulouse, bâti durant la première moitié du XVIII' siècle, est démoli vers 1907 lors du percement d'un tronçon du boulevard Raspail.

31. Le couvent, fondé en 1622, est détruit en 1897 pour laisser la place à la construction de l'hôtel de la Cour des comptes.

32. Lettre du 10 janvier. Autre jour de tristesse, le 14 avril, il conclut sa missive : " Je veux tout de même croire encore à ma chance, au Christ d'Amiens qui regarde l'avenir [...]... et qui tient un livre. [...] Oui, le Christ tient un livre, et le livre, un livre c'est toute la vie ! Combien je suis heureux de savoir que tu as fondé une bibliothèque ! un livre et une bonne action, les seules choses qui aient quelque prix!" 
pour les in-folio partout et mettre le pilastre en retrait ? Enfin, demande aux gens de l'art, et suis ton idée. Je crains les nids à poussière de ces retraits et que ce ne soit pas architectural. Voir la bibliothèque Mazarine et celle de l'Institut. " Madeleine s'empresse de modifier la commande à Ricci, l'entrepreneur menuisier, en lui précisant de fermer les portes de certains rayonnages par du fin grillage, toujours selon le goût de Champion.

\section{Le 17 avril 1916, Madeleine écrit à Pierre :}

Je viens de rentrer d'une promenade avec Jeanne. M. Rameau nous a emmenées à Sucy pour voir les grilles du château du Grand Val, que l'on morcelle, afin de nous rendre compte si l'une de ces grilles ne pourrait pas servir pour l'entrée de la bibliotheque. Je ne crois pas que cela fasse bien notre affaire; mais nous avons eu ainsi l'occasion de voir les restes d'un parc magnifique, dessiné au temps de Louis XVI, avec des bassins et des rivières dans le goût de Trianon. Quelle tristesse de voir tant de beaux arbres sur le point d'être abattus, des tas de cailloux autour d'un beau château, des murs éventrés, comme si la guerre avait passé par là. Ce morcellement a dû être commencé en 1912, par les Bernheim ${ }^{33}$, grands destructeurs de propriétés, en même temps que marchands de mauvaise peinture, confondue avec de bons tableaux.

Finalement pour la clôture sur la rue de la bibliothèque, «M. Rameau s'est occupé de chercher une grille qui nous manque; il a eu l'idée de demander aux propriétaires actuels du parc du Perreux une ancienne grille du parc, à moitié descellée, que l'on nous donnera en échange d'une banale grille neuve. Cette grille se compose de simples barreaux droits, en fer forgé, terminés par des pics; mais ces barreaux sont anciens et carrés " écrit Madeleine le 3 août, et encore le 6 août : "Je t'envoie des échantillons que M. Billaroud a fait pour la peinture des balcons. Le ton bleuâtre a été pris sur des balcons de Versailles, je crois qu'il s'harmoniserait très bien avec le ton de bois des bibliothèques; mais je ne veux pas me décider avant que tu m'aies donné ton avis. [...] M. Rameau a trouvé des volets de bois anciens qui, disposés horizontalement, pourraient former une cimaise."

Tout est dit des goûts du couple ; ils aiment "l'ancien " et quitte à donner à leur bibliothèque une allure classique, ils préferent réutiliser des éléments décoratifs des demeures d'antan plutôt que de faire réaliser des copies par des artisans contemporains.

\section{La fondation: une bibliotbèque exceptionnelle ${ }^{34}$}

Le bâtiment, désormais achevé, reste vide, jusqu'à la fin de la guerre. En avril et mai 1919, les collections d'Auguste sont transportées de Paris à Nogent. Pierre

33. Les fameux galeristes parisiens sont les marchands des néo-impressionnistes et des nabis; ils exposent aussi Van Gogh en 1901, Van Dongen en 1908, Matisse en 1910.

34. Sur l'histoire, l'architecture et le décor des bibliothèques françaises, voir André Masson, Le Décor des bibliotheques du Moyen Âge à la Révolution, Genève-Paris, librairie Droz, 1972. Histoire des bibliotheques franfaises, Paris, Promodis, éd. du Cercle de la Librairie, 4 vol. Les Bibliotheques parisiennes. Architecture et decor, Paris, Action artistique de la Ville de Paris, 2002. 
Champion adresse en décembre un rapport à l'administrateur général de la Bibliothèque nationale afin de lui exposer la manière dont il compte organiser la collection.

Comme les livres sont en partie déposés sur le plancher et en piles, [...] mon intention est de les mettre le plus tôt possible sur les rayons et de commencer leur classement. [...] J'estime que la collection peut comprendre environ 20000 volumes. Je viens de procéder au métrage des rayons qui donnerait un développement total de 750 mètres. [...] La fondation offre en effet un double caractère : elle doit être à la fois un musée et une bibliothèque publique. [...] Mon premier soin sera de faire descendre aux salles du rezde-chaussée, qui comprennent environ $\mathbf{2 5 0}$ mètres de rayons, les ouvrages de consultation courante, les dictionnaires, les ouvrages de littérature générale et d'histoire [...]. C'est en effet dans ces salles, où l'on accède par un escalier spécial de la rue, et qui sont séparées des autres pièces par des portes de fer, que la salle publique de lecture et de consultation sera établie. [...] Les salles du premier étage comprendront la bibliothèque-musée. Elles comportent des rayons, des armoires, un coffre-fort qui recevra les manuscrits, une galerie supérieure, où l'on accède par un escalier. Je conçois le classement et l'arrangement de ce premier étage comme une " réserve " où les livres seront mis en valeur de façon à présenter le meilleur aspect possible ${ }^{35}$.

Le rapport est approuvé en tous points et les époux Champion s'attellent à la tâche ${ }^{36}$.

Si aucune trace du programme donné à l'architecte ni aucun plan d'ensemble n'a pu être retrouvé, ce texte résume finalement assez bien le cahier des charges : un édifice aux fonctions triples, une collection de livres rares présentés précieusement, associée à un musée, et une bibliothèque publique. L'organisation spatiale doit, en outre, permettre une gestion distincte en deux pôles, avec des accès et des possibilités d'ouverture aux lecteurs séparés : la bibliothèque savante et la bibliothèque plus généraliste; l'ensemble sur $134 \mathrm{~m}^{2}$ au sol ! L'architecte superpose donc les deux bibliothèques, installant la salle publique dans un entre-sol afin de consacrer les espaces nobles à la bibliothèque-musée.

Dauphin traduit dans la pierre la volonté des commanditaires d'inscrire leur fondation dans l'espace urbain et conçoit l'ensemble comme un tout autonome, bien qu'en fait, ce bâtiment soit adossé aux deux demeures anciennes et encadré par les cours de celles-ci. Son entrée monumentale (celle qui conduit à la bibliothèque savante), tournée vers la ville et indépendante des deux maisons, confere au bâtiment une image d'édifice édilitaire; toutefois, on accède à la salle de lecture également depuis les maisons. Depuis la cour, un second escalier, plus discret, conduit à l'entresol. L'entrée principale ouvre sur un vestibule donnant accès à la grande salle de double hauteur en retour. Un escalier en tour demi hors ceuvre surmonté d'un tempietto articule les deux corps de bâtimentṣ en L. Le pavillon sur

35. Loc. cit., dossier E87/18.

36. Loc. cit., carton 42, correspondance entre les deux sceurs, 1914-1919. 
la rue, composé d'un rez-de-chaussée surélevé, d'un étage et d'un haut comble aménagé, accueille les vitrines, les meubles et les ouvres destinées au musée.

Répondant à la double vocation publique/privée de l'édifice, Dauphin choisit un style caractéristique de l'architecture domestique française de la fin du $\mathrm{XVI}^{e}$ siècle en brique et pierre, qu'il associe à la rigueur néoclassique du tempietto, de la composition architecturée de la porte ou de l'ordre monumental de la salle de lecture. Les élévations de cette dernière ne sont rythmées par aucune travée que des baies pourraient justifier puisque la lumière pénètre uniquement dans l'espace intérieur par un éclairage zénithal. Les murs pleins et lisses de cette salle, outre l'avantage d'accroître au maximum les linéaires de rayonnage à l'intérieur, accentuent le caractère précieux du contenu en renvoyant à l'idée du coffre-fort inviolable. La verrière est dissimulée derrière une balustrade en pierre qui évoque le toit terrasse ; ce corps de bâtiment cohabite étrangement avec le haut comble en pavillon, couvert d'ardoises, du second corps.

Durant les années 50, la rue Charles VII est élargie et la parcelle où s'implante la bibliothèque s'en trouve un peu rognée. La grille (le simple barreaudage réemployé du domaine du Perreux) qui clôturait une toute petite avant-cour est détruite et finalement remplacée par le haut mur visible actuellement, au nu du porche d'entrée. Le bâtiment ouvre désormais directement sur la rue.

De rares descriptions nous renseignent sur les intérieurs du musée (ill. 5) :

Ill. 5 : Vue intérieure du hall de la bibliothèque, espace réservé au musée, tirage photographique, vers 1920. BnF.

Le visiteur ne peut encore en voir qu'une partie embryonnaire, car il est loin d'être organisé ; il ne le sera que plus tard, lorsque les donateurs seront 
à même de joindre à la bibliothèque un certain nombre de pièces enclavées dans les bâtiments de leur habitation personnelle. Cependant, d'ores et déjà, dès son entrée et avant d'avoir mis le pied sur la première marche de marbre blanc, le visiteur peut voir à sa gauche une vitrine garnie de terres cuites et objets antiques [...]. Dans l'escalier tournant, une série de gravures, dessins ou tableaux encadrés ornant les murs [...]. Cette collection se continue dans la première salle du premier étage, où les murs sont garnis de dessins surtout relatifs au vieux Paris et d'un grand portrait à l'huile représentant le créateur de cette belle collection, $M$. Lesouëf. Dans la petite pièce du fond, se trouvent un grand Christ au tombeau de Henner, diverses toiles de cet artiste, dont deux portraits de Mme Pierre Champion, quelques autres toiles et deux vitrines contenant des miniatures, cires, éventails et autres objets, ainsi que deux autres vitrines contenant, l'une une collection de vieux ivoires japonais ou chinois, l'autre un choix de petits objets antiques, grecs ou égyptiens ${ }^{37}$.

La grande salle de la bibliothèque est, quant à elle, connue par diverses reproductions : les images prises par Jeanne et envoyées à son beau-frère au cours du chantier, photographies où les boiseries ne sont pas encore totalement installées, une reproduction accompagnant l'article de Léo Mouton ${ }^{38}$ de 1925 , des vues contemporaines de Champion (ill. 6), et d'autres encore datant d'après la seconde

\section{Illustration non autorisée à la diffusion}

III. 6 : Vue intérieure de la grande salle de la bibliothèque, tirage photographique, vers 1920 . BnF.

37. Léo Mouton, "La fondation Smith-Lesouëf et sa bibliothèque ", Revue des bibliothèques, $\mathrm{n}^{\text {os }}$ 7-10, 1926.

38. Le premier conservateur de la bibliothèque. 
guerre mondiale ${ }^{39}$. Ici, l'architecte s'efface devant les commanditaires qui choisissent avec soin chaque élément du décor. Pierre Champion, habitué des grandes bibliothèques savantes, se réfere clairement à ces modèles. Mais il connaît tout aussi bien d'autres temples du livre que sont les librairies et évoquera avec nostalgie, dans ses écrits, le souvenir de la première boutique de son père : "La librairie Champion, comme celle du père d'Anatole France, était une librairie à chaises. Les habitués y avaient leur place, comme à l'Académie on a son fauteuil " ${ }^{40}$ (ill. 7). Dans l'esprit

III. 7: Vue intérieure de la boutique d'Honoré Champion, lorsqu'elle était encore une "librairie à chaises ". Jacques Monfrin. op. cit., p. 2. Inventaire général. Cl. L. Kruszyk, ADAGP.

39. Musée de Nogent-sur-Marne.

40. Pierre Champion cité par Jacques Monfrin, op. cit., p. 32. 
de l'historien, malgré la richesse du fonds Smith-Lesouëf, il ne peut être question que d'installer le lecteur au coeur de la collection. À l'ouverture de la fondation, un bureau Mazarin trône au milieu de la pièce; il sera remplacé quelques années plus tard par une table pouvant accueillir conjointement plusieurs consultants.

Autour du lecteur, conformément aux usages, les rayonnages occupent toutes les surfaces murales de la salle, tel un vaisseau rectangulaire à double hauteur. Une galerie court sur son pourtour, disposition dont on trouve le premier exemple à la bibliothèque Ambrosienne de Milan en 1604 et qui devient habituelle au siècle suivant. Seule concession à la modernité, la lumière n'est plus obtenue par le rythme habituel de baies latérales, mais par un vaste éclairage zénithal. Ce parti, déjà adopté à la bibliothèque municipale d'Amiens en 1823, devient très fréquent au cours du $\mathrm{XIX}^{e}$ siècle. Toutefois, ici, la verrière demeure un strict élément fonctionnel, sans véritable parti décoratif.

L'attrait des commanditaires pour les choses "anciennes" se retrouve dans la présence en élévation de travées qui ne s'imposaient pas, en l'absence de fenêtre. Des pilastres ioniques rythment la composition des boiseries et déterminent les dimensions des armoires et des rayonnages. Champion insiste pour que les parties basses, réservées aux plus grands volumes, les in-folio, ne forment pas d'avant-corps saillant. À l'appui de sa demande, il évoque la bibliothèque de l'Institut, mais sa mémoire lui joue un tour, car il se réfere également au "modèle " que constitue la Mazarine, où les avant-corps y sont pourtant très marqués et forment des tablettes inclinées facilitant la consultation debout. Saillants ou non, comme ici, les rayonnages bas se doivent d'être soulignés, afin de faire office de soubassement à la composition architecturée de la bibliothèque classique.

Dernier élément du décor, la remarquable balustrade de la galerie. Composée de motifs de balustre stylisé contenu dans un cadre rectangulaire vertical, elle est caractéristique du travail de ferronnerie de la seconde moitié du $\mathrm{XVII}^{e}$ siècle. Les enroulements dans chacun des cadres évoquent parfaitement le piédouche, puis la panse et enfin le col d'un balustre. En outre, le centre de certains des motifs présente une croix latine surmontant un $\mathrm{M}$, pour Marie. Les panneaux réemployés du couvent parisien des Filles de l'Assomption formaient probablement à l'origine une clôture d'autel ou de chœur ${ }^{41}$.

Auguste Lesouëf achetait par passion personnelle et ne prit pas de dispositions pour assurer la pérennité de sa collection. C'est, finalement, par d'heureux concours de circonstance, que sa bibliothèque entra dans le domaine public et qu'un bâtiment fut spécifiquement construit pour l'accueillir. Les dons sont fréquents dans l'histoire des fonds publics français dont l'important réseau s'amorce à partir du $\mathrm{XIX}^{\mathrm{e}}$ siècle mais, le plus souvent, ils se composent uniquement de livres ou de manuscrits qui intègrent les locaux de l'établissement attributaire. Léguer une collection installée dans un édifice approprié semble faire figure d'exception; et le

41. La FNAGP conserve dans la bibliotheque des plans d'une balustrade signée du serrurier rouennais Ferdinand Marrou. Il s'agit probablement des dessins commandés par Dauphin et Marion qui ne furent pas réalisés. 
parti choisi par les Smith-Champion pour la fondation honorant leur oncle - un bâtiment de stature publique - encore moins fréquent. Les rares exemples franciliens connus l'attestent. Non loin de Nogent-sur-Marne, à Arcueil, Émile Raspail construit dans les années 1860 , une annexe à sa résidence. Le bâtiment, dont l'élévation évoque une façade d'église, permet à son père, François-Vincent (17941878) d'y accueillir ses réunions politiques, après son retour d'exil (ill. 8). À

IIl. 8: Maison d'Émile Raspail à Arcueil. Le bâtiment annexe, dédié à François-Vincent (l'inscription latine sur la façade "in patria carcer, laurus in exilio " [en prison dans sa patrie, des lauriers en exil] rappelle son destin politique), accueillit la bibliothèque de l'homme politique. Ensemble inscrit au titre des Monuments historiques en 1993. Inventaire général. Cl. C. Décamps, ADAGP.

la mort du grand homme, Émile rachète sa bibliothèque aux autres héritiers et installe la collection dans le bâtiment déjà dédié à son père. Mais, si son frère aîné, Benjamin, installé à Cachan dans une demeure d'Ancien Régime, offre sa maison et toutes les collections qu'il a religieusement conservées autour de l'œuvre paternelle, au département de la Seine, les propres héritiers d'Émile dispersent la bibliothèque Raspail en vente publique en $1912^{42}$. La maison et sa bibliothèque restent une résidence privée à la différence d'une autre collection fameuse, installée à

42. Ministère de la culture, direction régionale des affaires culturelles d'Île-de-France, dossiers des services des Monuments historiques et de l'Inventaire général. Archives départementales du Valde-Marnc, Alain Nafilyan (rééd.), Les Archives Raspail. Répertoire numérique de la sous-série $69 \mathrm{~J}$, Créteil, conscil général, 1994. 
Boulogne-Billancourt. Paul Marmottan (1856-1932), érudit passionné par l'histoire européenne au temps de Napoléon ${ }^{\mathrm{er}}$, installe sa bibliothèque, qu'il lègue à sa mort à l'Académie des beaux-arts, dans un bâtiment spécifique. Celui-ci, ainsi que sa maison d'habitation implantée non loin, sont réaménagés par ses soins dans le style Empire, entre 1890 et 1920 . Il ne cherche toutefois pas à y créer une atmosphère institutionnelle mais à reconstituer l'ambiance d'une demeure néo-classique (ill. 9) ${ }^{43}$.

Ill. 9 : Bibliothèque Paul-Marmottan, Boulogne-Billancourt. La bibliothèque vue du jardin. Ensemble inscrit au titre des Monuments historiques en 1984. Inventaire général. Cl. P. Ayrault, ADAGP.

Ainsi, en l'état actuel des connaissances, la bibliothèque de Nogent s'avère un édifice atypique qui cherche la synthèse impossible : initiative privée mais, dès l'origine, destinée au public ; bibliothèque d'érudit sur le modèle forgé durant l'Ancien Régime, qui tente de concilier le principe moderne de la bibliothèque généraliste d'accès libre ${ }^{44}$, bibliothèque et musée à la fois...

Dès 1945, selon le vœu des sœurs Smith, une maison de retraite pour des artistes est installée dans la bâtisse du 14, rue Charles VII. À partir de 1976, l'État réunit le legs Smith et celui d'Adèle de Rothschild ${ }^{45}$ pour constituer la fondation

43. Ministère de la culture, direction régionale des affaires culturelles d'île-de-France, dossiers des services des Monuments historiques et de l'Inventaire général. Bruno Foucart, « La bibliothèque Marmottan ", Connaissance des arts, $4^{c}$ trimestre 1994, p. 60-61.

44. Aussitôt élu maire, Champion créa une autre bibliothèque publique à Nogent, la bibliothèque municipale.

45. L'hôtel Salomon de Rothschild, rue Berryer à Paris, offert à l'État en 1922 pour en faire une « maison d'art $»$. 
nationale des arts graphiques et plastiques (FNAGP) qui désormais gère le domaine. Pour des raisons de conservation mais aussi de pertinence ${ }^{46}$, en 1980, la totalité de la collection Smith-Lesouëf est transférée à la Bibliothèque nationale, éclatée dans différents départements ${ }^{47}$, tandis que les objets de la donation Smith-Champion demeurent sur place ; le bâtiment-bibliothèque est mis à la disposition de la FNAGP. Aujourd'hui, cette dernière, après avoir construit des ateliers d'artiste dans le parc, rénové de fond en comble la maison de retraite, s'attèle désormais à un véritable projet de musée et d'espace d'exposition, pour les 14 bis et 16 , rue Charles VII, selon les dernières volontés des donatrices. Gageons qu'elle réussisse à donner une seconde vie à ce lieu dans lequel l'État n'a pas toujours su tenir tous ses engagements.

Isabelle DuHAu

Chercheur au service de l'inventaire général du patrimoine culturel à la direction régionale des affaires culturelles d'Ile-de-France

46. Les visiteurs se font si rares que la bibliothèque n'est ouverte que sur rendez-vous. En outre, le bâtiment, bien que classé en 1931 parmi les Bâtiments civils, n'est plus convenablement entretenu (loc. cit., dossier E87/15). Le portefeuille d'actions remis par les soeurs Smith en 1913 d'une valeur de 250000 francs est vendu en 1979. L'agent comptable de la Bibliothèque nationale est avisé par la caisse des dépôts et consignation que celle-ci vient de vendre les actions à leur valeur du jour, soit un montant de 3500 francs! (Loc. cit., E87/2).

47. En 1926, la collection numismatique avait dejà été rapportée à Paris ainsi que les manuscrits les plus précieux durant la seconde guerre mondiale, loc. cit., E87/2. 